\title{
Bifurcation analysis and chaotic behavior of a discrete-time delayed genetic oscillator model
}

\author{
Feng Liu ${ }^{1 *}$, Xiang Yin ${ }^{1}$, Fenglan Sun ${ }^{2}$, Xinmei Wang ${ }^{1}$ and Hua O Wang ${ }^{3}$
}

\footnotetext{
"Correspondence: Fliu@cug.edu.cn ${ }^{1}$ School of Automation, China University of Geosciences, Wuhan, 430074, P.R. China

Full list of author information is available at the end of the article
}

\begin{abstract}
In this paper, a genetic oscillator model with time delay is discretized by the Euler method. The discrete oscillator model is discussed by using Neimark-Sacker bifurcation theory. The direction and the stability of the Neimark-Sacker bifurcation has been studied using the center manifold theorem and normal form theory. Numerical simulations illustrate the theoretical results.
\end{abstract}

Keywords: stability; bifurcation; Euler discretized; genetic oscillator model

\section{Introduction}

In recent years, the dynamics of genetic oscillators has been investigated in the past decades [1-12]. Goldbeter shows that the genetic oscillators have a crucial impact on many aspects of cell physiology activities [3, 4]. Mathematical modeling has been playing key roles for understanding the dynamics of genetic oscillators [1-4]. The evolution of genetic oscillators can be described by ordinary differential equations or difference equations. Oscillation phenomena are common in nature, especially in those systems that have rhythm behavior. It has been demonstrated that one of the most common causes of the oscillation phenomenon is the existence of certain bifurcations [5-16]. In continuous-time systems, oscillations appear mainly due to Hopf bifurcations [8-11]. In discrete-time systems, however, the essential cause is Neimark-Sacker bifurcation or period-doubling bifurcation [13, $14]$.

Recently, increasing attention has been paid to discrete time models. The reasons are as follows: The numerical simulations of continuous-time models are obtained by the discretizing models. It is common practice to discretize the continuous-time model for experimental or computational purposes. The discrete-time model inherits the dynamic characteristics of the continuous-time model, and it also retains functional similarity to the continuous-time system and any physical or biological reality that the continuoustime model has [17]. At last, the discrete-time models have rich dynamical behaviors as compared to continuous-time models. We can get more accurate numerical simulations results from discrete time models. Herein, we will consider an oscillator model described by difference equations.

In this paper, we will investigate stability and Neimark-Sacker bifurcation of a discrete genetic oscillator model with delay. The genetic oscillator model [4] can be described by

(c) The Author(s) 2017. This article is distributed under the terms of the Creative Commons Attribution 4.0 International License (http://creativecommons.org/licenses/by/4.0/), which permits unrestricted use, distribution, and reproduction in any medium, provided you give appropriate credit to the original author(s) and the source, provide a link to the Creative Commons license, and indicate if changes were made. 
the differential equations:

$$
\left\{\begin{array}{l}
\frac{d X(t)}{d t}=k_{1} S \frac{K_{d}^{P}}{K_{d}^{P}+Y(t-\tau)^{P}}-k_{d x} X(t), \\
\frac{d Y(t)}{d t}=k_{s y} X(t)-k_{2} E_{T} \frac{Y(t)}{K_{m}+Y(t)},
\end{array}\right.
$$

where $X(t)$ and $Y(t)$ denote the concentration of mRNA and protein concentration at time $t$. $k_{1}$ and $k_{s y}$ represent the synthetic rate of mRNA and protein. $S$ is the concentration of a transcription factor. $K_{d}$ is the dissociation constant for binding of $Y(t)$. $P$ is Hill coefficient. $k_{d x}$ is the degradation rate of mRNA. $E_{T}$ is the total concentration of enzyme; its turnover rate is $k_{2}$ and its Michaelis constant is $K_{m} . \tau$ is the time delay required for transcription and translation.

This paper is organized as follows. In Section 2, the stability of the positive equilibrium and the existence of a Neimark-Sacker bifurcation are discussed. In Section 3, the direction and the stability of bifurcating periodic solutions can be determined by using the center manifold theorem and normal form theory. In Section 4, a simulation example is applied to verify the theoretical results. The last section contains conclusions.

\section{Stability analysis}

In order to be concise, let $P=2, n_{1}=k_{1} S, n_{2}=K_{d}^{2}, n_{3}=k_{d x}, n_{4}=k_{s y}, n_{5}=k_{2} E_{T}, n_{6}=K_{m}$, then equation (1.1) can be rewritten as

$$
\left\{\begin{array}{l}
\frac{d X(t)}{d t}=\frac{n_{1} n_{2}}{n_{2}+Y(t-\tau)^{2}}-n_{3} X(t), \\
\frac{d Y(t)}{d t}=n_{4} X(t)-n_{5} \frac{Y(t)}{n_{6}+Y(t)} .
\end{array}\right.
$$

Let $\tilde{x}(t)=X(\tau t), \tilde{y}(t)=Y(\tau t)$, then equation (2.1) can be rewritten as

$$
\left\{\begin{array}{l}
\dot{\tilde{x}}(t)=\frac{\tau n_{1} n_{2}}{n_{2}+\tilde{y}(t-1)^{2}}-\tau n_{3} \tilde{x}(t), \\
\dot{\tilde{y}}(t)=\tau n_{4} \tilde{x}(t)-\tau n_{5} \frac{\tilde{y}(t)}{n_{6}+\tilde{y}(t)} .
\end{array}\right.
$$

Applying the forward Euler scheme to equation (2.2), we obtain a model as follows:

$$
\left\{\begin{array}{l}
\tilde{x}(n+1)=\left(1-\tau h n_{3}\right) \tilde{x}(n)+\frac{\tau h n_{1} n_{2}}{n_{2}+\tilde{y}(n-d)^{2}}, \\
\tilde{y}(n+1)=\tau h n_{4} \tilde{x}(n)+\tilde{y}(n)-\tau h n_{5} \frac{\tilde{y}(n)}{n_{6}+\tilde{y}(n)} .
\end{array}\right.
$$

Here $h=1 / d$ is the step size, $d$ is a positive integer.

In the following sections, we will study the dynamics behavior of the system (2.3) including stability and the bifurcation phenomenon. We will use some lemmas to illuminate existence conditions of a unique positive root of equation (2.4).

We denote $(x, y)$ as the steady state and $\left(x^{*}, y^{*}\right)$ as the positive equilibrium point of equation (2.3). Let $(x, y)$ be a steady state of equation (2.3), then we have

$$
\left\{\begin{array}{l}
\frac{n_{1} n_{2}}{n_{2}+y^{2}}-n_{3} x=0, \\
n_{4} x-n_{5} \frac{y}{n_{6}+y}=0 .
\end{array}\right.
$$


From equation (2.4), we obtain

$$
y^{3}+M y-N=0
$$

where $M=\frac{n_{2} n_{3} n_{5}-n_{1} n_{2} n_{4}}{n_{3} n_{5}}, N=\frac{n_{1} n_{2} n_{4} n_{6}}{n_{3} n_{5}}$.

Let $f(y)=y^{3}+M y-N$.

Taking the derivative of $f(y)$, we have

$$
f^{\prime}(y)=3 y^{2}+M
$$

Lemma 2.1 System (2.3) has a unique positive equilibrium point $\left(x^{*}, y^{*}\right)$ if one of the following assumptions holds:

(i) $0<n_{1} n_{4} / n_{3} n_{5}<1$;

(ii) $n_{1} n_{4} / n_{3} n_{5}>1$ and $f\left(r_{1}\right)<0$;

(iii) $n_{1} n_{4} / n_{3} n_{5}>1, f\left(r_{1}\right)>0$, and $f\left(r_{2}\right)>0$,

where $f(y)=y^{3}+M y-N, r_{1}$ and $r_{2}$ are roots of $f^{\prime}(y)=0$.

Proof As $f(y)=y^{3}+M y-N$. Since $f(0)=-N<0, f(+\infty)=+\infty$, it is clear that equation (2.5) has at least one positive solution.

We will consider three cases as follows:

If (i) holds, then $\Delta=-12 M \leq 0$.

This means that $f^{\prime}(y) \geq 0$. Hence $f(y)$ is monotonically increasing on $[0,+\infty)$. Based on the above analysis, we know that the positive root is unique.

If (ii) holds, it means that $\Delta=-12 M>0$, then $f^{\prime}(y)=0$ has two roots:

$$
r_{1}=\frac{-\sqrt{-3 M}}{3}<0, \quad r_{2}=\frac{\sqrt{-3 M}}{3}>0
$$

We obtain $r_{1}<0, r_{2}>0$ and $r_{1}<r_{2}$, then $f(y)$ is monotonically increasing on $\left(-\infty, r_{1}\right)$ and $\left[r_{2},+\infty\right)$, or decreasing on $\left[r_{1}, r_{2}\right)$, this together with $f\left(r_{1}\right)<0$ imply that $f(y)$ has a unique positive root.

Similarly, if (iii) holds, $f(y)$ has a unique positive root.

It means that under one of the assumptions (i)-(iii), there is a unique positive equilibrium point $\left(x^{*}, y^{*}\right)$ in the system (2.3). This completes the proof.

Let $u(n)=\tilde{x}(n)-x^{*}, v(n)=\tilde{y}(n)-y^{*}$. Substituting them into equation (2.3), we have

$$
\left\{\begin{array}{l}
u(n+1)=\left(1-\tau h n_{3}\right) u(n)+\frac{\tau h n_{1} n_{2}}{n_{2}+\left(v(n-d)+y^{*}\right)^{2}}-\tau h n_{3} x^{*} \\
v(n+1)=\tau h n_{4}\left(u(n)+x^{*}\right)+v(n)-\tau h n_{5} \frac{v(n)+y^{*}}{n_{6}+\left(v(n)+y^{*}\right)}
\end{array}\right.
$$

Introducing a variable $X(n)=(u(n), v(n), v(n-1), \ldots, v(n-d))^{T}$, equation $(2.5)$ can be transformed into the following form:

$$
X(n+1)=F(X(n), \tau)
$$


where $F=\left(F_{0}, F_{1}, \ldots, F_{d}\right)^{T}$. We have

$$
F_{k}= \begin{cases}\left(1-\tau h n_{3}\right) u(n)+\frac{\tau h n_{1} n_{2}}{n_{2}+\left(v(n-d)+y^{*}\right)^{2}}-\tau h n_{3} x^{*}, & k=0, \\ \tau h n_{4}\left(u(n)+x^{*}\right)+v(n)-\tau h n_{5} \frac{v(n)+y^{*}}{n_{6}+\left(v(n)+y^{*}\right)}, & k=0, \\ u(n-k+1), & 1 \leq k \leq m, \\ v(n-k+1), & 1 \leq k \leq m .\end{cases}
$$

Then we can get the linear part of equation (2.6) as follows:

$$
X(n+1)=A X(n)
$$

where

$$
A=\left[\begin{array}{ccccc}
1-\tau h n_{3} & 0 & \cdots & 0 & \frac{-2 \tau h n_{1} n_{2} *^{*}}{\left(n_{2}+y^{* 2}\right)^{2}} \\
\tau h n_{4} & 1-\frac{\tau h n_{5} n_{6}}{\left(n_{6}+y^{*}\right)} & \cdots & 0 & 0 \\
0 & 1 & \cdots & 0 & 0 \\
\vdots & \vdots & \ddots & \vdots & \vdots \\
0 & 0 & \cdots & 1 & 0
\end{array}\right]_{(d+2) \times(d+2)}
$$

The characteristic equation of equation (2.7) is given by

$$
\lambda^{d+2}+a_{1} \lambda^{d+1}+a_{2} \lambda^{d}+a_{3}=0
$$

where

$$
\begin{aligned}
& a_{1}=\left(\tau h b_{1}+\tau h n_{3}\right)-2, \quad a_{2}=\left(1-\tau h b_{1}\right)\left(1-\tau h n_{3}\right), \quad a_{3}=2 \tau^{2} h^{2} b_{2}, \\
& b_{1}=\frac{n_{5} n_{6}}{\left(n_{6}+y^{*}\right)^{2}}, \quad b_{2}=\frac{n_{1} n_{2} n_{4} y^{*}}{\left(n_{2}+y^{* 2}\right)^{2}} .
\end{aligned}
$$

According to the knowledge of the dynamics, we know that the equilibrium stability of equation (2.6) is determined by the roots distribution of equation (2.9). We will utilize a lemma of Zhang et al. [18] to analyze the roots distribution of equation (2.9).

Lemma 2.2 There exists a $\bar{\tau}>0$ such that, for $0<\tau<\bar{\tau}$, all roots of equation (2.9) have modulus less than one.

Proof When $\tau=0$, we can obtain $a_{1}=-2, a_{2}=1, a_{3}=0$.

Then equation (2.9) becomes

$$
\lambda^{d+2}-2 \lambda^{d+1}+\lambda^{d}=0
$$

The equation has $d$-fold roots $\lambda=0$, and two-fold roots $\lambda=1$.

Consider the root $\lambda(\tau)$ such that $|\lambda(0)|=1$. This root depends continuously on $\tau$ and is a differentiable function of $\tau$. 
Differentiating both sides of equation (2.9) with respect to $\tau$ yields

$$
(d+2) \lambda^{d+1} \lambda^{\prime}+a_{1}(d+1) \lambda^{d} \lambda^{\prime}+a_{1}^{\prime} \lambda^{d+1}+a_{2}^{\prime} \lambda^{d}+a_{2} d \lambda^{d-1} \lambda^{\prime}+a_{3}^{\prime}=0,
$$

where $\lambda^{\prime}=\frac{d \lambda}{d \tau}, a_{1}^{\prime}=\frac{d a_{1}}{d \tau}=h\left(b_{1}+n_{3}\right), a_{2}^{\prime}=\frac{d a_{2}}{d \tau}=2 \tau h^{2} b_{1} n_{3}-h\left(b_{1}+n_{3}\right), a_{3}^{\prime}=\frac{d a_{3}}{d \tau}=4 \tau h^{2} b_{2}$.

Differentiating both sides of equation (2.10) with respect to $\tau$ yields

$$
\begin{aligned}
& (d+2)\left[(d+1) \lambda^{d}\left(\lambda^{\prime}\right)^{2}+\lambda^{d+1} \lambda^{\prime \prime}\right] \\
& \quad+(d+1)\left[a_{1}^{\prime} \lambda^{d} \lambda^{\prime}+a_{1} \lambda^{d} \lambda^{\prime \prime}+a_{1} d \lambda^{d-1}\left(\lambda^{\prime}\right)^{2}\right]+a_{1}^{\prime}(d+1) \lambda^{d} \lambda^{\prime} \\
& \quad+a_{2}^{\prime \prime} \lambda^{d}+a_{2}^{\prime} d \lambda^{d-1} \lambda^{\prime}+d\left[a_{2}^{\prime} \lambda^{d-1} \lambda^{\prime}+a_{2}(d-1) \lambda^{d-2}\left(\lambda^{\prime}\right)^{2}+a_{2} \lambda^{d-1} \lambda^{\prime \prime}\right]+a_{3}^{\prime \prime}=0 .
\end{aligned}
$$

From equation (2.11), we obtain

$$
\begin{gathered}
{\left[(d+2) \lambda^{d+1}+a_{1}(d+1) \lambda^{d}+a_{2} d \lambda^{d-1}\right] \lambda^{\prime \prime}+\left[(d+2)(d+1) \lambda^{d}+a_{1}(d+1) d \lambda^{d-1}\right.} \\
\left.+\left[2(d+1) a_{1}^{\prime} \lambda^{d}+a_{2} d(d-1) \lambda^{d-2}\right]\left(\lambda^{\prime}\right)^{2}+2 a_{2}^{\prime} d \lambda^{d-1}\right] \lambda^{\prime}+a_{2}^{\prime \prime} \lambda^{d}+a_{3}^{\prime \prime}=0
\end{gathered}
$$

Substituting $\lambda=1, \tau=0$ into equation (2.12), we have

$$
\left(\lambda^{\prime}\right)^{2}+h\left(b_{1}+n_{3}\right) \lambda^{\prime}+2 h^{2} b_{2}+h^{2} b_{1} n_{3}=0
$$

Solving equation (2.13), we have

$$
\left.\frac{d \lambda}{d \tau}\right|_{\tau=0, \lambda=1}=-h b_{1}+i h \sqrt{2 b_{2}} \text { or }\left.\frac{d \lambda}{d \tau}\right|_{\tau=0, \lambda=1}=-h n_{3}+i h \sqrt{2 b_{2}} .
$$

By calculation, we have

$$
\begin{aligned}
& \left.\frac{d|\lambda|^{2}}{d \tau}\right|_{\tau=0, \lambda=1}=\left.\left[\lambda \frac{d \lambda^{-}}{d \tau}+\lambda^{-} \frac{d \lambda}{d \tau}\right]\right|_{\tau=0, \lambda=1}=\left.2 \operatorname{Re}\left(\lambda^{-} \frac{d \lambda}{d \tau}\right)\right|_{\tau=0, \lambda=1}, \\
& \left.\frac{d|\lambda|^{2}}{d \tau}\right|_{\tau=0, \lambda=1}=-2 h n_{3}<0, \quad \text { or }\left.\quad \frac{d|\lambda|^{2}}{d \tau}\right|_{\tau=0, \lambda=1}=-2 h b_{1}<0 .
\end{aligned}
$$

Consequently, $|\lambda|<1$ holds for all sufficiently small $\tau>0$. Thus, all roots of equation (2.9) lie in $|z|<1$ for sufficiently small positive $\tau$, and existence of the maximal $\bar{\tau}$ follows. This completes the proof.

Denote by $e^{i \omega^{*}}$ a root of equation (2.9) at $\tau=\tau^{*}$, then we have

$$
e^{i(d+2) \omega^{*}}+a_{1}^{*} e^{i(d+1) \omega^{*}}+a_{2}^{*} e^{i d \omega^{*}}+a_{3}^{*}=0,
$$

in which $a_{1}^{*}, a_{2}^{*}, a_{3}^{*}$ can be obtained by substituting $\tau^{*}$ into $a_{1}, a_{2}, a_{3}$. Separating the real and imaginary parts, we have

$$
\begin{aligned}
& a_{2}^{*} \cos d \omega^{*}+a_{1}^{*} \cos (d+1) \omega^{*}+\cos (d+2) \omega^{*}=-a_{3}^{*}, \\
& a_{2}^{*} \sin d \omega^{*}+a_{1}^{*} \sin (d+1) \omega^{*}+\sin (d+2) \omega^{*}=0 .
\end{aligned}
$$


So

$$
\cos \left(\omega^{*}\right)=\frac{1}{16 a_{2}^{*}\left( \pm \sqrt{\Delta_{1}}-a_{1}^{*} a_{2}^{*}-a_{1}^{*}\right)}
$$

where $\Delta_{1}=a_{1}^{* 2}\left(a_{2}^{*}-1\right)^{2}-4 a_{2}^{*}\left(1-2 a_{2}^{*}+a_{2}^{* 2}-a_{3}^{* 2}\right)$,

$$
a_{1}^{*}=\tau^{*} h\left(b_{1}+n_{3}\right)-2, \quad a_{2}^{*}=\left(1-\tau^{*} h b_{1}\right)\left(1-\tau^{*} h n_{3}\right), \quad a_{3}^{*}=2 \tau^{* 2} h^{2} b_{2} .
$$

Lemma 2.3 Assume the step size $h$ is sufficiently small. If $\Delta_{1} \geq 0$, and $0<16 a_{2}^{*}\left(-a_{1}^{*} a_{2}^{*}-\right.$ $\left.a_{1}^{*} \pm \sqrt{\Delta_{1}}\right)<1$, then equation (2.9) has no root with modulus one for all $\tau>0$.

Proof Assume that the step size $h$ is sufficiently small.

For sufficiently small $h>0$, if $\Delta_{1} \geq 0$, and $0<16 a_{2}^{*}\left(-a_{1}^{*} a_{2}^{*}-a_{1}^{*} \pm \sqrt{\Delta_{1}}\right)<1$, then we obtain $\cos \omega^{*}>1$. This is a contradiction. So we complete proof.

From Lemma 2.3, we know that if $16 a_{2}^{*}\left(-a_{1}^{*} a_{2}^{*}-a_{1}^{*} \pm \sqrt{\Delta_{1}}\right)>1$, then the roots $e^{ \pm i \omega^{*}}$ of (2.9) with modulus one satisfy

$$
\begin{aligned}
& \cos \left(\omega^{*}\right)=\frac{1}{16 a_{2}^{*}\left( \pm \sqrt{\Delta_{1}}-a_{1}^{*} a_{2}^{*}-a_{1}^{*}\right)}, \\
& \tau^{*}=\frac{\left(b_{1}+n_{3}\right)\left(\sin (d+1) \omega^{*}-\sin d \omega^{*}\right)+\sqrt{\Delta_{2}}}{2 h b_{1} n_{3} \sin d \omega^{*}}, \quad h=1 / d .
\end{aligned}
$$

Here $\Delta_{1}=a_{1}^{* 2}\left(a_{2}^{*}-1\right)^{2}-4 a_{2}^{*}\left(1-2 a_{2}^{*}+a_{2}^{* 2}-a_{3}^{* 2}\right)$

$$
\Delta_{2}=\left(b_{1}+n_{3}\right)^{2}\left(\sin (d+1) \omega^{*}-\sin d \omega^{*}\right)^{2}-4 b_{1} n_{3} \sin d \omega^{*}\left(\sin (d+2) \omega^{*}+\sin d \omega^{*}\right) .
$$

It is easy to see that there is a sequence of time delays $\tau_{0}<\tau_{1}<\tau_{2}<\cdots<\tau_{n}$ satisfies equation (2.14).

Lemma 2.4 The inequality $d_{h}=\left.\frac{d|\lambda|^{2}}{d \tau}\right|_{\lambda=\lambda^{*}, \tau=\tau^{*}}>0$ holds for sufficiently small $h$.

Proof For sufficiently small $h$, we have

$$
\begin{aligned}
d_{h}= & \left.\frac{d|\lambda|^{2}}{d \tau}\right|_{\tau=\tau^{*}, \omega=\omega^{*}}=\left.\left[\lambda^{-} \frac{d \lambda}{d \tau}+\lambda \frac{d \lambda^{-}}{d \tau}\right]\right|_{\tau=\tau^{*}, \lambda=\lambda^{*}} \\
= & -\left[\lambda^{-} \frac{h\left(b_{1}+n_{3}\right) \lambda^{d+1}+\left(2 h^{2} b_{1} n_{3}-h\left(b_{1}+n_{3}\right)\right) \lambda^{d}+4 \tau h^{2} b_{2}}{(d+2) \lambda^{d+1}+(d+1) \lambda^{d} a_{1}+d \lambda^{d-1} a_{2}}\right. \\
& \left.+\lambda \frac{h\left(b_{1}+n_{3}\right) \bar{\lambda}^{d+1}+\left(2 h^{2} b_{1} n_{3}-h\left(b_{1}+n_{3}\right)\right) \bar{\lambda}^{d}+4 \tau h^{2} b_{2}}{(d+2) \bar{\lambda}^{d+1}+(d+1) \bar{\lambda}^{d} a_{1}+d \bar{\lambda}^{d-1} a_{2}}\right] \\
= & \frac{h\left(b_{1}+n_{3}\right)\left(3 d+2+2 \cos \omega^{*}\right)}{S} .
\end{aligned}
$$

Here

$$
\begin{aligned}
S= & {\left[(d+2)^{2}+(d+1)^{2} a_{1}^{2}+d^{2} a_{2}^{2}\right] } \\
& +2\left[(d+1)(d+2) a_{1}+(d+1) d a_{1} a_{2}\right] \cos \omega^{*}+2(d+2) d a_{2} \cos 2 \omega^{*} .
\end{aligned}
$$


Note that

$$
\begin{aligned}
{\left[(d+2)^{2}+(d+1)^{2} a_{1}^{2}+d^{2} a_{2}^{2}\right] } & \\
& +2\left[(d+1)(d+2) a_{1}+(d+1) d a_{1} a_{2}\right] \cos \omega^{*}+2(d+2) d a_{2} \cos 2 \omega^{*} \\
< & {\left[(d+2)^{2}+(d+1)^{2} a_{1}^{2}+d^{2} a_{2}^{2}\right] } \\
& +2\left[(d+1)(d+2) a_{1}+(d+1) d a_{1} a_{2}\right]+2(d+2) d a_{2} \\
= & {\left[(d+2)+(d+1) a_{1}+d a_{2}\right]^{2}, } \\
0< & \frac{h\left(b_{1}+n_{3}\right)\left(3 d+2+2 \cos \omega^{*}\right)}{\left[(d+2)+(d+1) a_{1}+d a_{2}\right]^{2}} \leq d_{h} .
\end{aligned}
$$

This completes the proof.

From Lemmas 2.1-2.4, we obtain the following theorem.

Theorem 2.1 When the parameters of system (2.3) are defined, we have:

(i) When $\tau \in\left[0, \tau_{0}\right)$, the equilibrium $\left(x^{*}, y^{*}\right)$ of system (2.3) is asymptotically stable.

When $\tau>\tau_{0}$, the equilibrium $\left(x^{*}, y^{*}\right)$ of system (2.3) is unstable.

(ii) When $\tau=\tau_{0}$, for the equilibrium $\left(x^{*}, y^{*}\right)$ of system (2.3) there will exist a

Neimark-Sacker bifurcation. This is to say, for system (2.3) there exists a cluster of periodic solutions bifurcating near the equilibrium at $\tau=\tau_{0}$.

Remark According to the above discussions and applying the Neimark-Sacker bifurcation theory presented in Kuznetsov [19], we obtain Theorem 2.1. We can see that the stability of the equilibrium $\left(x^{*}, y^{*}\right)$ varies as the parameter $\tau$ varies. It is shown that the equilibrium is asymptotically stable for $\tau \in\left[0, \tau_{0}\right)$, and unstable for $\tau>\tau_{0}$. We can observe that the Neimark-Sacker bifurcation occurs when the time delay crosses the critical value $\tau_{0}$. According to the results of Theorem 2.1, we can see that the conclusions of the discrete system (2.3) are consistent with those of the continuous models (see $[8,10,11])$.

\section{Direction and stability of the Neimark-Sacker bifurcation}

In this section, we will use bifurcation theory [19-21] to discuss the direction and stability of the Neimark-Sacker bifurcation of system (2.3) for considering the delay time as a bifurcation parameter.

Let $\tau^{*}$ is the critical value $\tau_{j}(j=0,1,2, \ldots)$ of the origin, at which system (2.6) undergoes a Neimark-Sacker bifurcation.

If it is sufficiently smooth, equation (2.6) can be transformed into the form

$$
X_{n+1}=A X_{n}+\frac{1}{2} B\left(X_{n}, X_{n}\right)+\frac{1}{6} C\left(X_{n}, X_{n}, X_{n}\right)+o\left(\left\|X_{n}\right\|^{4}\right),
$$

where

$$
\begin{aligned}
& B\left(X_{n}, X_{n}\right)=\left(B_{1}\left(X_{n}, X_{n}\right), B_{2}\left(X_{n}, X_{n}\right), \ldots, B_{d+2}\left(X_{n}, X_{n}\right)\right)^{T}, \\
& C\left(X_{n}, X_{n}, X_{n}\right)=\left(C_{1}\left(X_{n}, X_{n}, X_{n}\right), C_{2}\left(X_{n}, X_{n}, X_{n}\right), \ldots, C_{d+2}\left(X_{n}, X_{n}, X_{n}\right)\right)^{T},
\end{aligned}
$$


and

$$
\begin{aligned}
& B_{1}\left(X_{n}, X_{n}\right)=\frac{2 \tau h n_{1} n_{2}\left(2 y^{* 2}-1\right)}{\left(n_{2}+y^{* 2}\right)^{2}} v_{n-d}^{2}, \\
& C_{1}\left(X_{n}, X_{n}, X_{n}\right)=\frac{4 \tau h n_{1} n_{2} y^{*}\left(5-4 y^{* 2}\right)}{\left(n_{2}+y^{* 2}\right)^{4}} v_{n-d}^{3}, \\
& B_{k}=C_{k}=0, \quad 2 \leq k \leq d+2 .
\end{aligned}
$$

Lemma 3.1 If $q=\left(q_{1}, q_{2}, q_{3}, q_{4}, \ldots, q_{d+2}\right)^{T}$ is the eigenvector of $A$ and its eigenvalue is $e^{i \omega^{*}}$; $p=\left(p_{1}, p_{2}, \ldots, p_{d+2}\right)^{T}$ is the eigenvector of $A^{T}$, and its eigenvalue is $e^{-i \omega^{*}}$, then there exist

$$
q=\left[\begin{array}{c}
1 \\
\frac{\tau^{*} h n_{4}}{e^{i \omega^{*}+\tau^{*} h b_{1-1}}} \\
\left(\frac{\tau^{*} h n_{4} e^{-i \omega^{*}}}{e^{i \omega^{*}}+\tau^{*} h b_{1}-1}\right) \\
\vdots \\
\left(\frac{\tau^{*} h n_{4} e^{-i \omega^{*} n}}{e^{i \omega^{*}}+\tau^{*} h b_{1}-1}\right)
\end{array}\right], \quad p=\left[\begin{array}{c}
p_{1} \\
\frac{\tau^{*} h n_{4}}{e^{-i \omega^{*}}+\tau^{*} h n_{3}-1} \\
\frac{\tau^{*} h n_{4}\left(e^{-i \omega^{*}}+\tau^{*} h b_{1}-1\right)}{e^{-i \omega^{*}}+\tau^{*} h n_{3}-1} \\
\vdots \\
\frac{\tau^{*} h n_{4} e^{-i(d-1) \omega^{*}}\left(e^{-i \omega^{*}}+\tau^{*} h b_{1}-1\right)}{e^{-i \omega^{*}}+\tau^{*} h n_{3}-1}
\end{array}\right] .
$$

Proof Let $q\left(\tau^{*}\right) \in C^{d+2}$ be a complex eigenvector of $A\left(\tau^{*}\right)$ corresponding to $e^{i \omega^{*}}, p\left(\tau^{*}\right) \in$ $C^{d+2}$ be a complex eigenvector of transposed matrix $A^{T}\left(\tau^{*}\right)$ corresponding to $e^{-i \omega^{*}}$, then we have

$$
\begin{array}{ll}
A\left(\tau^{*}\right) \bar{q}\left(\tau^{*}\right)=e^{-i \omega^{*}} \bar{q}\left(\tau^{*}\right), & A\left(\tau^{*}\right) q\left(\tau^{*}\right)=e^{i \omega^{*}} q\left(\tau^{*}\right), \\
A^{T}\left(\tau^{*}\right) p\left(\tau^{*}\right)=e^{-i \omega^{*}} p\left(\tau^{*}\right), & A^{T}\left(\tau^{*}\right) \bar{p}\left(\tau^{*}\right)=e^{i \omega^{*}} \bar{p}\left(\tau^{*}\right) .
\end{array}
$$

It is noted that $q\left(\tau^{*}\right), p\left(\tau^{*}\right)$ should satisfy $\langle p, q\rangle=1$, where $\langle p, q\rangle=\sum_{j=0}^{d} \bar{p}_{j} q_{j}$.

Let $q=\left(q_{1}, q_{2}, \ldots, q_{d+2}\right)^{T}$ and $p=\left(p_{1}, p_{2}, \ldots, p_{d+2}\right)^{T}$.

Let $q_{1}=1$, then we can get the eigenvector $q$.

It can be obtained following by calculating:

$$
\begin{aligned}
& q_{2}=\frac{\tau^{*} h n_{4}}{e^{i \omega^{*}}+\tau^{*} h b_{1}-1} q_{1}, \\
& q_{d+2}=-\frac{2 \tau^{*} h n_{1} n_{2} y^{*}}{\left(e^{i \omega^{*}}+\tau^{*} h n_{3}-1\right)\left(n_{2}+y^{* 2}\right)^{2}} q_{1}, \quad \text { and } q_{j+1}=e^{-i \omega^{*}} q_{j}, j=2,3, \ldots, d+1 .
\end{aligned}
$$

Then $p$ can be obtained in the same way:

$$
\begin{aligned}
& p_{2}=\frac{\tau^{*} h n_{4}}{e^{-i \omega^{*}}+\tau^{*} h n_{3}-1} p_{1}, \\
& p_{3}=\frac{\tau^{*} h n_{4}\left(e^{-i \omega^{*}}+\tau^{*} h b_{1}-1\right)}{e^{-i \omega^{*}}+\tau^{*} h n_{3}-1} p_{1}, \quad \text { and } \quad p_{j+1}=e^{-i \omega^{*}} p_{j}, j=4,5, \ldots, d+1 .
\end{aligned}
$$

Then the proof of Lemma 3.1 is complete.

Let a real two dimensional eigenspace of $e^{ \pm \omega^{*} j}$ be $T^{c}$, and $T^{s}$ be a $d$ dimensional eigenspace which is other than $T^{c}$. 
Take any $x \in R^{d+2}$, whose decomposition is

$$
x=z q+\bar{z} \bar{q}+y, \quad z \in C, z q+\bar{z} \bar{q} \in T^{C}, y \in T^{S} .
$$

Regarding the variable $z$ as a coordinate on $T^{C}$,

$$
y=-\langle p, x\rangle q-\langle\bar{p}, x\rangle \bar{q}+x, \quad z=\langle p, x\rangle .
$$

So, we have a format of the map $F$ at $\tau=\tau^{*}$ in the above coordinate as follows:

$$
\begin{aligned}
& z \mapsto e^{j \omega^{*}} z+\langle p, F(z q+\bar{z} \bar{q}+y)\rangle, \\
& y \mapsto A y+F(z q+\bar{z} \bar{q}+y)-\langle p-F(z q+\bar{z} \bar{q}+y)\rangle q-\langle\bar{p}, F(z q+\bar{z} \bar{q}+y)\rangle \bar{q} .
\end{aligned}
$$

Then we can obtain the following formulas by applying Taylor expansions:

$$
\begin{aligned}
& z \mapsto e^{i \omega^{*}} z+\frac{1}{2} g_{20} z^{2}+g_{11} z \bar{z}+\frac{1}{2} g_{02} \bar{z}^{2}+\frac{1}{2} g_{21} z^{2} \bar{z}+\left\langle G_{10}, y\right\rangle c z+\left\langle G_{01}, y\right\rangle \bar{z} \\
& y \mapsto A y+\frac{1}{2} H_{20} z^{2}+H_{11} z \bar{z}+\frac{1}{2} H_{02} \bar{z}^{2}+o\left(|z|^{3}\right) .
\end{aligned}
$$

Here $g_{i j} \in C, G_{10}, G_{01} \in C^{d+2}$, and

$$
\begin{aligned}
& g_{11}=\langle p, B(q, \bar{q})\rangle, \quad g_{02}=\langle p, B(\bar{q}, \bar{q})\rangle, \\
& g_{20}=\langle p, B(q, q)\rangle, \quad g_{21}=\langle p, C(q, y)\rangle, \\
& \left\langle G_{01}, y\right\rangle=\langle p, B(\bar{q}, y)\rangle, \quad\left\langle G_{10}, y\right\rangle=\langle p, B(q, y)\rangle, \\
& H_{20}=B(q, q)-\langle p, B(q, q)\rangle q-\langle\bar{p}, B(q, q)\rangle \bar{q}, \\
& H_{11}=B(q, \bar{q})-\langle p, B(q, \bar{q})| q-\langle\bar{p}, B(q, \bar{q})\rangle \bar{q} \\
& H_{02}=B(\bar{q}, \bar{q})-\langle p, B(q, \bar{q})\rangle q-\langle\bar{p}, B(\bar{q}, \bar{q})| \bar{q} .
\end{aligned}
$$

So, we can obtain the format of the center manifold

$$
y=V(z, \bar{z})=\frac{1}{2} w_{20} z^{2}+w_{11} z \bar{z}+\frac{1}{2} w_{20} \bar{z}^{2}+o\left(|z|^{3}\right)
$$

where $\left\langle q^{*}, w_{i j}\right\rangle=0$.

Putting equation (3.2) into equation (3.1), then we have

$$
\left\{\begin{array}{l}
w_{20}=\left(e^{2 i \omega^{*}} I-A\right)^{-1} H_{20}, \\
w_{11}=(I-A)^{-1} H_{11}, \\
w_{02}=\left(e^{-2 i \omega^{*}} I-A\right)^{-1} H_{02} .
\end{array}\right.
$$

We can obtain the Taylor coefficients as follows:

$$
\begin{aligned}
& g_{11}=\langle p, B(q, \bar{q})\rangle, \quad g_{02}=\langle p, B(\bar{q}, \bar{q})\rangle, \quad g_{20}=\langle p, B(q, q)\rangle, \\
& g_{21}=\langle p, C(q, q, \bar{q})\rangle-2\left\langle p, B\left(q,(I-A)^{-1} B(q, \bar{q})\right)\right\rangle+\left\langle p, B\left(\bar{q},\left(e^{2 i \omega^{*}} I-A\right)^{-1} B(q, q)\right)\right\rangle
\end{aligned}
$$




$$
\begin{aligned}
& +\frac{e^{-i \omega^{*}}\left(1-e^{2 i \omega^{*}}\right)}{1-e^{-i \omega^{*}}}\langle p, B(q, q)\rangle\langle p, B(q, \bar{q})\rangle \\
& -\frac{2}{1-e^{-i \omega^{*}}}|\langle p, B(\bar{q}, \bar{q})\rangle|^{2}-\frac{e^{i \omega^{*}}}{e^{3 i \omega^{*}}-1}|\langle p, B(\bar{q}, \bar{q})\rangle|^{2} .
\end{aligned}
$$

Define

$$
c_{1}(\tau)=\frac{g_{20} g_{11}(\bar{z}-3+2 z)}{2\left(z^{2}-2\right)(\bar{z}-1)}+\frac{\left|g_{11}\right|^{2}}{1-\bar{z}}+\frac{\left|g_{02}\right|^{2}}{2\left(z^{2}-\bar{z}\right)}+\frac{g_{21}}{2} .
$$

It easy to obtain $c_{1}\left(\tau^{*}\right)$ by substituting $z=e^{-i \omega^{*}}$ into equation (3.3).

Theorem 3.1 When $l>0(<0)$, where $l=-\operatorname{Re}\left[e^{i \omega^{*}} c_{1}\left(\tau^{*}\right)\right] / d_{h}$, we know that if the curve exists for $\tau>\tau^{*}$, the bifurcation of equation (2.4) is supercritical (subcritical); when $\operatorname{Re}\left[e^{-i \omega^{*}} c_{1}\left(\tau^{*}\right)\right]<0$, or $\operatorname{Re}\left[e^{-i \omega^{*}} c_{1}\left(\tau^{*}\right)\right]>0$ the bifurcation is orbitally stable or unstable.

\section{Numerical simulation}

To illustrate the analytical results obtained, we consider the following particular case of equation (2.5). Let $n_{1}=0.7, n_{2}=0.1, n_{3}=0.1, n_{4}=1, n_{5}=1, n_{6}=0.2, h=0.2, d=5$. Then $\tau_{0} \approx 1.4667$ is the critical value such that Neimark-Sacker bifurcation occurs. Figures 1-5 are the waveform plots and phase diagrams of equation (2.5).

In Figure 1, we show that the waveform plot and phase diagram for equation (2.5) when $\tau=1<\tau_{0}$ and for the equilibrium $\left(x^{*}, y^{*}\right)$ are asymptotically stable.

In Figure 2, we show that the waveform plot and phase diagram for equation (2.5) when $\tau=1.467>\tau_{0}$ and near $\tau_{0}\left(x^{*}, y^{*}\right)$ are unstable. From Theorems 2.1 and 3.1, there is a Neimark-Sacker bifurcation.

In Figure 3, we show that the waveform plot and phase diagram for equation (2.5) when $\tau=1.5>\tau_{0}$ and $\left(x^{*}, y^{*}\right)$ are unstable. There are multiple Neimark-Sacker bifurcations.

In Figure 4, we show that the waveform plot and phase diagram for equation (2.5) when $\tau=2>\tau_{0}$ and $\left(x^{*}, y^{*}\right)$ are unstable. There is a Neimark-Sacker bifurcation.

In Figure 5, we show that for the waveform plot and phase diagram for equation (2.5) when $\tau=4.1>\tau_{0}$, the system (2.5) appears to show chaotic behavior. Figures $5(\mathrm{c})$ and (d)

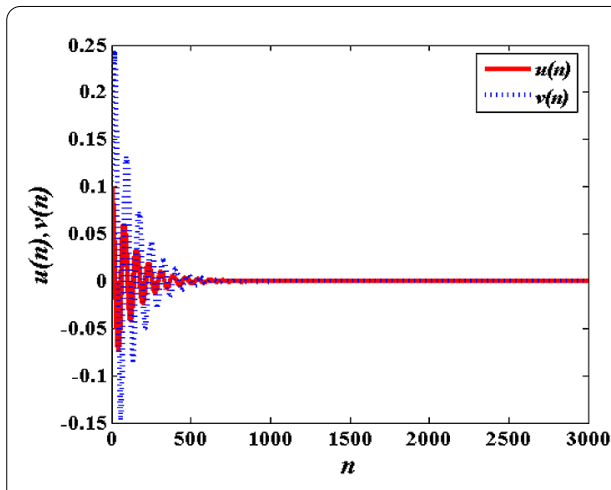

(a)

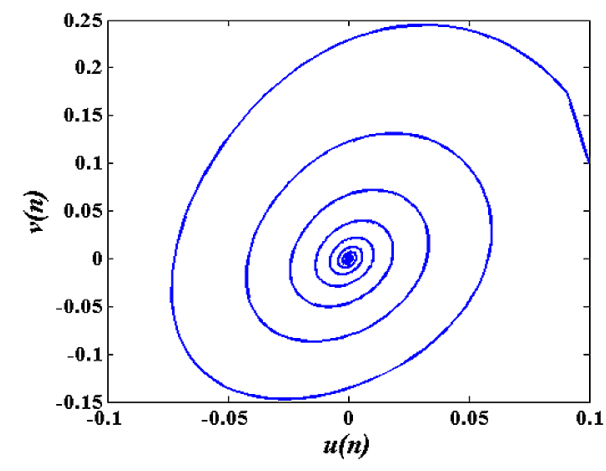

(b)

Figure 1 Numerical simulations of equation (2.5) for $\tau=1<\tau_{0}$. (a) The waveform plot of equation (2.5) with $\tau=1<\tau_{0}$; (b) the phase diagram of equation (2.5) with $\tau=1<\tau_{0}$. 


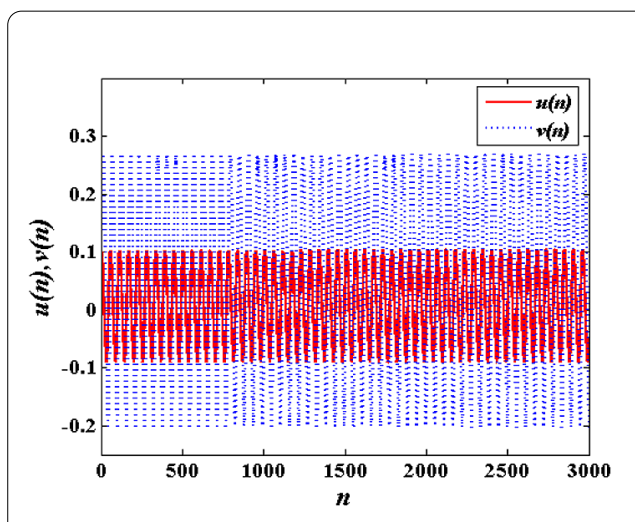

(a)

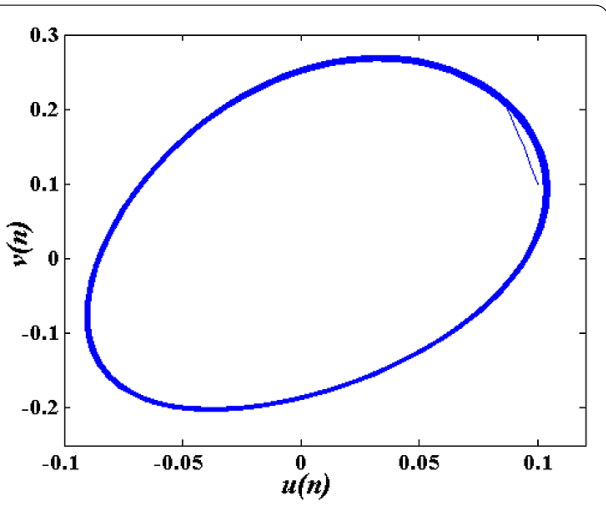

(b)

Figure 2 Numerical simulations of equation (2.5) for $\boldsymbol{\tau}=1.467>\tau_{0}$. (a) The waveform plot of equation (2.5) with $\tau=1.467>\tau_{0}$; (b) the phase diagram of equation (2.5) with $\tau=1.467>\tau_{0}$.

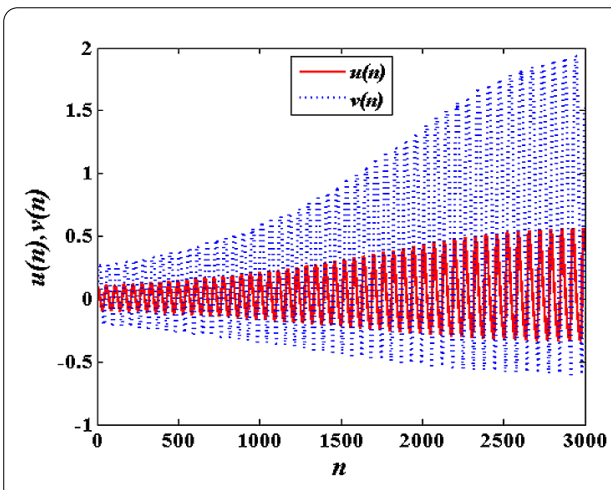

(a)

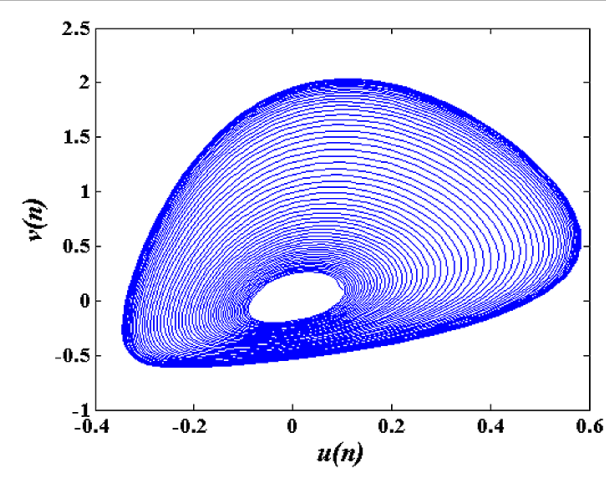

(b)

Figure 3 Numerical simulations of equation (2.5) for $\boldsymbol{\tau}=\mathbf{1 . 5}>\boldsymbol{\tau}_{\mathbf{0}}$. (a) The waveform plot of equation (2.5) with $\tau=1.5>\tau_{0}$; (b) the phase diagram of equation (2.5) with $\tau=1.5>\tau_{0}$.

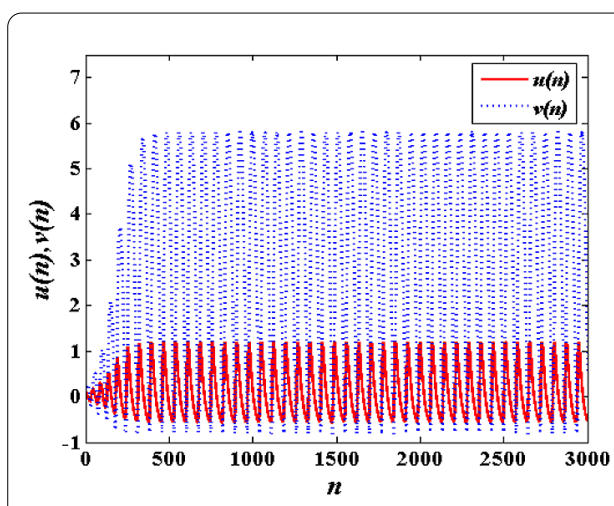

(a)

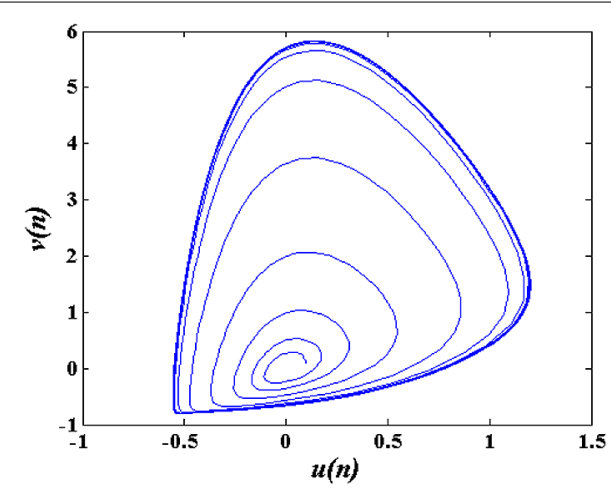

(b)

Figure 4 Numerical simulations of equation (2.5) for $\boldsymbol{\tau}=\mathbf{2}>\boldsymbol{\tau}_{0}$. (a) The waveform plot of equation (2.5) with $\tau=2>\tau_{0}$; (b) the phase diagram of equation (2.5) with $\tau=2>\tau_{0}$. 


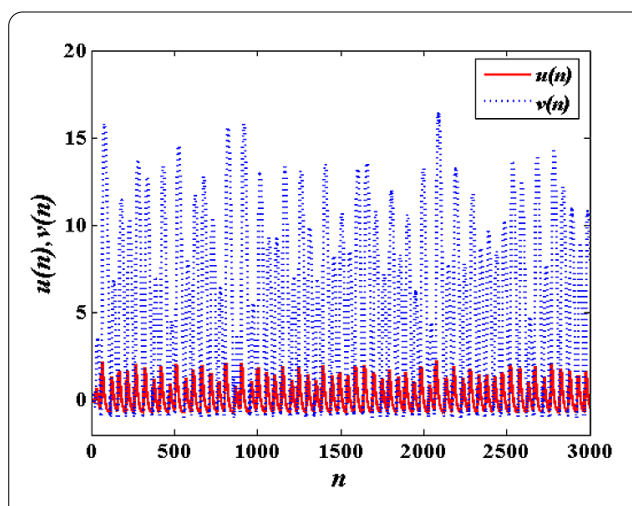

(a)

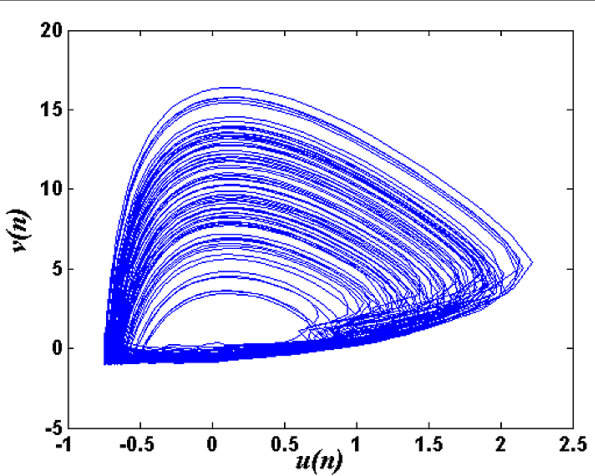

(b)

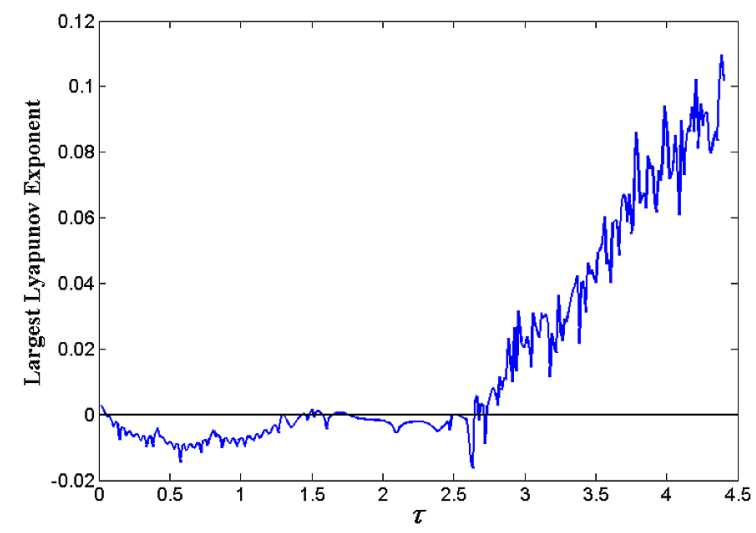

(c)

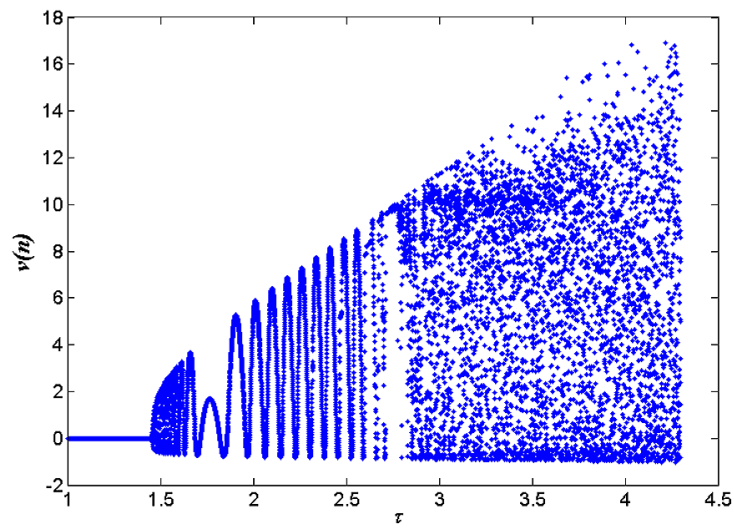

(d)

Figure 5 Numerical simulations of equation (2.5) for $\boldsymbol{\tau}=\mathbf{4 . 1}>\boldsymbol{\tau}_{\mathbf{0}}$. (a) The waveform plot of equation (2.5) with $\tau=4.1>\tau_{0}$; (b) the phase diagram of equation (2.5) with $\tau=4.1>\tau_{0}$; (c) the largest Lyapunov spectrum for system (2.5); (d) bifurcation diagram for system (2.5), in the $(\tau, v)$-plane, for $\tau \in[1,4.4]$. 
present the bifurcation diagram and the Lyapunov spectrum for system (2.5), for $\tau \in$ $[1,4.4]$. Figure 5 presents the complex behavior of system (2.5) in a neighborhood of the origin, for $\tau \in[1,4.4]$, revealing the routes to chaos starting from the Neimark-Sacker and other bifurcations.

From Figures 1-5, we can observe that a Neimark-Sacker bifurcation occurs when the time delay crosses the critical value $\tau_{0}$, It is shown that the positive equilibrium is asymptotically stable for $\tau \in\left[0, \tau_{0}\right)$, and unstable for $\tau>\tau_{0}$ which is entirely consistent with the results in Theorem 2.1. It can be found that there are different kinds of Neimark-Sacker bifurcations for different time delays and when the system undergoes a variety of bifurcations, for the system can be found the chaos phenomenon. Chaos has important significance to the life system. It shows that the parameter time delay has important influence on the dynamic behaviors of the system, which may easily generate complex oscillations and chaos.

\title{
5 Conclusions
}

A discrete-time genetic network with delay was considered in this paper. Some sufficient conditions of local stability of equilibrium points were given. By choosing the time delay as a bifurcation parameter, we show that the Neimark-Sacker bifurcation would occur when the bifurcation parameter crosses some critical values. We obtained a formula for determining the direction and stability of a Neimark-Sacker bifurcation.

The theoretical studies on discrete-time genetic network model may not only contribute to the understanding of dynamic relation among different elements.

Competing interests
The authors declare that they have no competing interests.

Authors' contributions

$\mathrm{FL}$ and $\mathrm{XY}$ wrote the first draft of a paper and did revising and editing; all authors were in charge of the choice of the topic, the method, revising, and editing. All authors read and approved the final manuscript.

\begin{abstract}
Author details
${ }^{1}$ School of Automation, China University of Geosciences, Wuhan, 430074, P.R. China. ${ }^{2}$ Research Center of System Theory and Application, Chongqing University of Posts and Telecommunications, Chongqing, 400065, P.R. China. ${ }^{3}$ Department of Mechanical Engineering, Boston University, Boston, MA 02215, USA.
\end{abstract}

\section{Acknowledgements}

The authors are very grateful to the editor and anonymous referees for their valuable comments and helpful suggestions, which have led to a great improvement of the original manuscript. This work was partially supported by National Natural Science Foundation (NNSF) of China under Grant 61472374, 61503053, 61672112, 11401110, 61603358, the fund of research center for advanced control of complex systems \& intelligent geosciences instrument, China University of Geosciences (Wuhan) (AU2016CJ021).

Received: 22 May 2016 Accepted: 2 December 2016 Published online: 03 January 2017

References

1. Hidde, DJ: Modeling and simulation of genetic regulatory systems: a literature review. J. Comput. Biol. 9, 67-103 (2002)

2. Kobayashi, T, Chen, L, Aihara, K: Modeling genetic switches with positive feedback loops. J. Theor. Biol. 221, 379-399 (2003)

3. Goldbeter, A: Computational approaches to cellular rhythms. Nature 420, 238-245 (2002)

4. Tyson, JJ: Design principles of biochemical oscillators. Nat. Rev. Mol. Cell Biol. 9, 981-982 (2008)

5. Chen, L, Aihara, K: Stability of genetic regulatory networks with time delay. IEEE Trans. Biomed. Circuits Syst. 49, 602-608 (2002)

6. Li, C, Chen, L, Aihara, K: Stability of genetic networks with sum regulatory logic: Lur'e system and LMI approach. IEEE Trans. Biomed. Circuits Syst. 53(11), 2451-2458 (2006)

7. He, W, Cao, J: Robust stability of genetic regulatory networks with distributed delay. Cogn. Neurodyn. 2, 355-361 (2008)

8. Wan, A, Zou, X: Hopf bifurcation analysis for a model of genetic regulatory system with delay. J. Math. Anal. Appl. 356, 464-476 (2009) 
9. Shen, J, Liu, Z, Zheng, W, Xu, F, Chen, L: Oscillatory dynamics in a simple gene regulatory network mediated by small RNAs. Physica A 388, 2995-3000 (2009)

10. Wang, K, Wang, L, Jiang, H, Teng, Z: Stability and bifurcation of genetic regulatory networks with delays. Neurocomputing 73, 2882-2892 (2010)

11. Xiao, M, Zheng, W, Cao, J: Stability and bifurcation of genetic regulatory networks with small RNAs and multiple delays. Int. J. Comput. Math. 91, 5907-5927 (2014)

12. Wan, $X, X u, L$, Fang, H, Yang, F, Li, X: Exponential synchronization of switched genetic oscillators with time-varying delays. J. Franklin Inst. 351, 4395-4414 (2014)

13. Jiang, XW, Zhan, XS, Guan, ZH, Zhang, XH, Yu, L: Neimark-Sacker bifurcation analysis on a numerical discretization of Gause-type predator-prey model with delay. J. Franklin Inst. 352(1), 1-15 (2015)

14. He, X, Li, CD, Huang, TW, Yu, JZ: Bifurcation behaviors of an Euler discretized inertial delayed neuron model. Sci. China, Technol. Sci. 59, 418-427 (2016)

15. Wang, B, Jian, J: Stability and Hopf bifurcation analysis on a four-neuron BAM neural network with distributed delays. Commun. Nonlinear Sci. Numer. Simul. 15, 189-204 (2010)

16. Yu, P, Lin, W: Complex dynamics in biological systems arising from multiple limit cycle bifurcation. J. Biol. Dyn. 10, 263-285 (2016)

17. Mohamad, S, Naim, A: Discrete-time analogues of integro-differential equations modelling bidirectional neural networks. J. Comput. Appl. Math. 138, 1-20 (2002)

18. Zhang, C, Liu, M, Zheng, B: Hopf bifurcation in numerical approximation of a class delay differential equations. Appl. Math. Comput. 146, 335-349 (2003)

19. Kuznetsov, YA: Elements of Applied Bifurcation Theory. Springer, New York (1998)

20. Hassard, BD, Kazarinoff, ND, Wan, YH: Theory and Applications of Hopf Bifurcation. Cambridge University Press, Cambridge (1981)

21. Han, M, Yu, P: Normal Forms, Melnikov Functions, and Bifurcations of Limit Cycles. Springer, New York (2012)

\section{Submit your manuscript to a SpringerOpen ${ }^{\ominus}$ journal and benefit from:}

- Convenient online submission

Rigorous peer review

- Immediate publication on acceptance

- Open access: articles freely available online

- High visibility within the field

- Retaining the copyright to your article 